\title{
Besonderer Fokus
}

\section{auf junge Zahnärzte hat imponiert}

\begin{abstract}
"Ich möchte Neuheiten begutachten - den State-of-the-art der Zahnheilkunde inklusive aller aktuellen Innovationen auf einen Blick. Dazu interessante Leute treffen, auch Bekannte wiedersehen, Gespräche führen und meinen Horizont erweitern", so lauteten die generellen Erwartungen angehender junger Zahnärzte an die Internationale Dental-Schau (IDS). Das klingt ganz ähnlich wie bei vielen "alten Hasen“, die schon zum zehnten oder zwanzigsten Mal dabei waren. Und doch erlebten Zahnmedizinstudenten, Berufseinsteiger und Kollegen in den ersten Jahren nach ihrer Niederlassung die IDS 2017 etwas anders ..
\end{abstract}

Christian Ehrensberger // Frankfurt am Main

$\mathrm{M}$ anche der jungen Besucher nahmen sich einen, manche vier oder fünf Tage Zeit für die Messe. Tendenziell legten sie besonders hohen Wert auf den Themenkreis rund um „digitale Workflows, 3D-Röntgen, CAD/CAM“, denn in der Altersgruppe zwischen 20 und 30 Jahren ist man sich heute bewusst, dass dies die eigene berufliche Laufbahn deutlich stärker beeinflussen wird als vorherige Generationen.

Die Atmosphäre in den Messehallen haben die angehenden und „frischgebackenen“ Zahnärztinnen und Zahnärzte durchweg als positiv empfunden, manche sogar als „euphorisch“. Wer zum ersten Mal zur IDS reiste, fühlte sich zuweilen auch etwas überfordert von der schieren Menge der Informationen. Spätestens am vierten Tag stieß die Aufnahmekapazität des eigenen Gehirns an ihre Grenzen.

\section{Lust auf intensiven Austausch zu Innovationen}

An praktisch allen Messeständen fühlten sich die jüngeren Besucher mit offenen Armen empfangen. Das Klischee eines „typischen Vertreters“, der vor allen Dingen am Verkauf interessiert ist, fand sich nirgendwo - stattdessen überall gut geschulte und freundliche Fachleute mit Lust auf intensive Gespräche zu neuen Verfahren und innovativen Produkten.

Was dem einen oder anderen „Newcomer“ aufgefallen ist: Manche Hersteller, die er zum Beispiel als Spezialisten für Scanner kennengelernt hat, bieten jetzt auch Röntgengeräte oder 3D-Drucker. Vieles wächst hier zusammen und führt zum

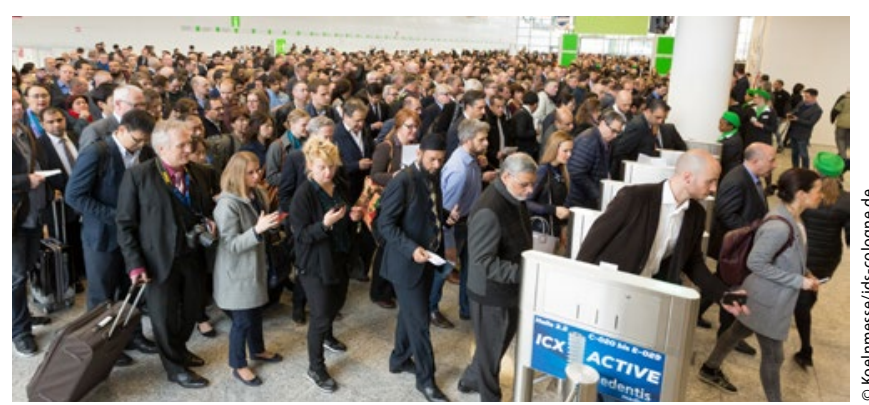

Die größte IDS aller Zeiten: Über 155.000 Besucher strömten in die Messehallen.
Angebot weitreichender Prozessketten. Diese stießen bei jungen Zahnärzten auf besonderes Interesse, denn es stellt sich doch die Frage: Wie arbeite ich in der Praxis mit reinen Chairside-Systemen? Wie setzt sich der digitale Workflow ins zahntechnische Labor fort? Wie sehen die Schnittstellen aus? Auf diese und viele andere Fragen gab die IDS Antworten in Form von neuen CAD/ CAM-Verfahren, innovativen Druckern oder Software-Updates.

\section{"Career Day“ und "Generation Lounge“ öffnen Türen}

Ausgesprochen positiv registrierten junge Zahnärzte die vielfältigen Fortbildungsmöglichkeiten, wie sie von immer mehr Unternehmen angeboten werden. Manche begleiten Studierende bis zum Eintritt in die Selbstständigkeit und darüber hinaus.

Der „Career Day“ am Donnerstag der IDS zeigte berufliche Entwicklungsmöglichkeiten alternativ oder zusätzlich zur Niederlassung auf. Dabei nahmen die jungen Zahnärzte gern die Gelegenheit zu persönlichen Gesprächen in der sogenannten „Recruitment Lounge“ wahr. Warum nicht eine Karriere in Forschung oder Vertrieb eines Unternehmens der Dentalindustrie in Erwägung ziehen? Oder als Zahnärztin oder Zahnarzt Produkte vor ihrer Einführung im „Feldtest“ auf Herz und Nieren prüfen oder selbst Fortbildungen zu neuen Therapieverfahren geben?

Insgesamt hatten die jungen Besucher den Eindruck, dass sich die ausstellenden Unternehmen zunehmend auf sie fokussierten. Das fanden sie beeindruckend!

Über die Stände der Unternehmen der Dentalindustrie hinaus zeigten sich gerade junge Besucher von den vielfältigen Angeboten der Verbände angetan. So informierten sich viele am Stand der Bundeszahnärztekammer über aktuelle Themen, wie die zahnmedizinische Behandlung von Geflüchteten oder über Möglichkeiten zum sozialen und gesellschaftlichen Engagement beim BZÄK-Kooperationspartner HDZ (Stiftung Hilfswerk Deutscher Zahnärzte für Lepra- und Notgebiete) oder zum Arbeiten als Zahnarzt im Ausland.

$\mathrm{Zu}$ großer Beliebtheit brachte es auch die Generation Lounge. Junge Zahnärzte schätzten hier insbesondere die Präsenz des BdZA (Bundesverband der zahnmedizinischen Alumni). Sie nutzten die Lounge, um so manchen interessanten Kontakt zu knüpfen. Auf diese Weise entstanden womöglich viele persönliche Netzwerke, bleibende Konstanten für ein ganzes Berufsleben. 